\title{
INTEGRAL ESTIMATES OF CONFORMAL METRICS
}

\author{
XINGWANG XU \\ (Communicated by Peter Li )
}

\begin{abstract}
In this article we show that there exists a rational number $\mu$, depending only on the dimension $n(\geq 5)$ of the manifold such that the $\mu$ thpower of the conformal factor is bounded in $\mathrm{H}_{2}^{2}$ norm in terms of volume bound and the square norm bound of the scalar curvature of the conformal metrics. Some applications are also given.
\end{abstract}

\section{INTRODUCTION}

Let $\left(M, g_{0}\right)$ be a compact connected oriented Riemannian manifold. According to the solution of the well-known Yamabe problem, we can assume that the scalar curvature of the metric $g_{0}$ is a constant. We are going to show in this paper the following

Theorem 1. Let $\left(M, g_{0}\right)$ be as above. Let $g=u^{\frac{4}{n-2}} g_{0}$ be a new metric with conformal factor $u$. Assume $n \geq 5$ and set $\mu=\frac{n-4}{(n-2)}$. If $\int_{M} s_{g}^{2} d v_{g} \leq C_{0}$ and $\int_{M} u^{\frac{2 n}{n-2}} d v_{0} \leq C_{1}$, there exists a constant $C=C\left(C_{0}, C_{1}, g_{0}\right)>0$ such that $\left\|u^{\mu}\right\|_{H_{2}^{2}} \leq C$.

Recently there has been increasing interest in compactness theorems for Riemannian manifolds under various geometric assumptions (see, among others, [GLP], $[\mathrm{GW}],[\mathrm{P}],[\mathrm{Ga1}, 2])$. More recently, it has been found that the boundedness conditions on the curvature as in $[\mathrm{GLP}],[\mathrm{GW}]$ and $[\mathrm{P}]$ can be replaced by some integral bounds of the full curvature tensors [Ga1,2]. One of the often used integral bounds is the $L^{p}$ bound on the curvature tensor, with $p \geq \frac{n}{2}$, where $n$ is real dimension of the underlying manifolds. For instance, in [Ga1] and [Mo], the authors show that if $\left\{\left(M_{i}, g_{i}\right)\right\}$ is a sequence of Einstein manifolds of real dimension $2 n$ satisfying

(i) $\operatorname{Diam}\left(M_{i}, g_{i}\right) \leq \mu$,

(ii) $\int_{M}\left\|R m\left(g_{i}\right)\right\|_{g_{i}}^{n} d v_{g_{i}} \leq \mu$ and

(iii) $\operatorname{Vol}\left(M_{i}, g_{i}\right) \geq \frac{1}{\mu}$,

where $\mu$ is a uniform constant, then the subsequence of $\left\{\left(M_{i}, g_{i}\right)\right\}$ converges to an Einstein orbifold with finitely many isolated singular points.

On the other hand, M. Gromov points out that any compact manifold carries Riemannian metrics of volume 1 with $L^{p}$-norm of the curvature tensor as small as you like for $p<\frac{n}{2}$. Our Theorem 1 shows that if we restrict the metrics in the same

Received by the editors June 22, 1994.

1991 Mathematics Subject Classification. Primary 58G25, 53C21.

(C)1996 American Mathematical Society 
conformal class, those metrics are not so wild in a certain sense. Of course, it is clear that one can not expect to have $C^{0}$ compactness without further assumptions.

Our Theorem 1 also should be compared with M. Gursky's results. He used the $L^{p}$ bound of the full curvature tensors to control $H_{2}^{2}$ norm of the conformal factor with $p>\frac{n}{2}$. Of course, his results are stronger than ours even in dimension 5 or 6 . But his assumption is much stronger than ours.

The second motivation for us to prove Theorem 1 is the compactness of the isospectral family of conformal metrics. In [CY1,2],[BPY],[G],[An], they show that for dimension 3 , the isospectral family of conformal metrics is $C^{\infty}$ compact. As M. Anderson points out, in order to get $C^{\infty}$ compactness for isospectral metrics of Riemannian manifolds of dimension three, we only need to assume that the Sobolev constants have uniform lower bound; it is not necessary to restrict metrics in the same conformal class. Dimension three is special because $2>\frac{3}{2}$; by the above compactness theorem, we only need to have $L^{2}$ bounds on the full curvature tensors, which is already contained in the heat invariants.

Notice that this $L^{2}$ bound of the full curvature tensors from spectrum is not only true for three dimensional manifolds; it is also true for manifolds of dimension less than six. Dimension four is the delicate case since $L^{2}$ norm of the full curvature tensor is topological invariant. In [BCY], T. Branson, A. Chang and P. Yang consider dimension four with respect to comformal Laplace operator. They show that in terms of log determinent of conformal Laplace, the $H_{2}^{2}$ norm of conformal factor can be bounded for a 4-dimensional compact symmetric Einstein manifold which is neither the standard 4-sphere nor a hyperbolic space. In $[\mathrm{X}]$, we show that the isospectral family of conformal metrics of the negative conformal class is compact in $C^{\infty}$ topology if $L^{4}$ norm of the scalar curvatures of new conformal metrics is bounded. For the standard 4-sphere, up to conformal group, the same statement holds.

When we look at higher dimensional manifolds, we obtain as a corollary the following

Theorem 2. If the dimension $n$ of the Riemannian manifolds is 5 or 6 , and if $\left\{u_{i}^{\frac{4}{n-2}} g_{0}\right\}$ are conformal metrics which are isospectral with respect to the Laplace operator, then $\left\{u_{i}^{\frac{n-4}{(n-2)}}\right\}$ is a (pre)compact set in $H_{1}^{2}$ where $M$ is any compact, orientable connected manifold and $g_{0}$ is any Riemannian metric with constant scalar curvature.

The idea for the proof of Theorem 1 is to use the curvature assumption and the relationship between new curvature tensors and the background one to get the bound we want. Of course, we have to be more careful in order to use the integration by parts technique. For the proof of Theorem 2, it is standard to apply heat invariants to bound the square norm of scalar curvatures, thus reduce it to Theorem 1. The proof will be given in $\S 3$ after we have set down Theorem 1 in $\S 2$.

\section{Proof of Theorem 1}

Let $\left(M, g_{0}\right)$ be a compact Riemannian manifold with Riemannian metric $g_{0}$. Consider the new metric $g=u^{\frac{4}{n-2}} g_{0}$ for a smooth positive function $u$ on $M$. Then the relation of scalar curvatures between two metrics is known to be

$$
s_{g}=u^{-\frac{n+2}{n-2}}\left(s_{0} u-c_{n} \Delta u\right)
$$


where $\Delta$ denotes the Laplace operator with respect to the metric $g_{0}$ and $c_{n}=$ $\frac{4(n-1)}{n-2}$.

By a well-known theorem, we can and will assume that $g_{0}$ has constant scalar curvature $s_{0}$. Our starting point is the following

Lemma 1. If $n \geq 5, \int_{M} s_{g}^{2} d v_{g} \leq C_{0}$ and $\int_{M} u^{\frac{2 n}{n-2}} d v_{0} \leq C_{1}$, then there is a positive constant $C_{2}>0$ depending on $C_{0}, C_{1}$ and the geometry of the manifold $\left(M, g_{0}\right)$ such that

$$
\int_{M}(\Delta u)^{2} u^{1-p} d v_{0} \leq C_{2}
$$

where $p=\frac{n+2}{n-2}$.

Proof.

$$
\begin{aligned}
\int_{M} s_{g}^{2} d v_{g}= & \int_{M} u^{\frac{-2(n+2)}{n-2}}\left(s_{0} u-c_{n} \Delta u\right)^{2} u^{\frac{2 n}{n-2}} d v_{0} \\
= & \int_{M} u^{\frac{-4}{n-2}}\left[s_{0}^{2} u^{2}-2 c_{n} s_{0} u \Delta u+c_{n}^{2}(\Delta u)^{2}\right] d v_{0} \\
= & \int_{M} s_{0}^{2} u^{\frac{2(n-4)}{n-2}} d v_{0}-2 c_{n} s_{0} \int_{M} u^{\frac{n-6}{n-2}}(\Delta u) d v_{0}+c_{n}^{2} \int_{M} \frac{(\Delta u)^{2}}{u^{\frac{4}{n-2}}} d v_{0} \\
\geq & \int_{M} s_{0}^{2} u^{\frac{2(n-4)}{n-2}} d v_{0}-\left\{\epsilon c_{n}^{2} \int_{M} \frac{(\Delta u)^{2}}{u^{\frac{4}{n-2}}} d v_{0}+\frac{1}{\epsilon} \int_{M} s_{0}^{2} u^{\frac{2(n-4)}{n-2}} d v_{0}\right\} \\
& +c_{n}^{2} \int_{M} \frac{(\Delta u)^{2}}{u^{\frac{4}{n-2}}} d v_{0} .
\end{aligned}
$$

From (3) and by choosing $\epsilon=\frac{1}{2}$ we can easily see that

$$
\begin{aligned}
c_{n}^{2} \int_{M} \frac{(\Delta u)^{2}}{u^{\frac{4}{n-2}}} d v_{0} & \leq 2 \int_{M} s_{g}^{2} d v_{g}+2 \int_{M} s_{0}^{2} u^{\frac{2(n-4)}{n-2}} d v_{0} \\
& \leq 2 C_{0}+2 s_{0}^{2} \int_{M} u^{\frac{2(n-4)}{n-2}} d v_{0} \\
& \leq 2 C_{0}+2 s_{0}^{2}\left\{\int_{M} u^{\frac{2 n}{n-2}} d v_{0}\right\}^{\frac{n-4}{n}}\left\{\int_{M} d v_{0}\right\}^{\frac{4}{n}} \\
& \leq 2 C_{0}+2 s_{0}^{2} C_{1}^{\frac{n-4}{n}}(\operatorname{vol}(M))^{\frac{4}{n}} \\
& \equiv C_{2} c_{n}^{2} .
\end{aligned}
$$

Since $p-1=\frac{4}{n-2}$, the proof of Lemma 1 is completed.

Let us denote the traceless Ricci tensor for the metric $g_{0}$ by $B_{0 i j}$. By the wellknown Weitzenbock formula, we have

Lemma 2.

$$
\begin{aligned}
\int_{M}\left(\sum u_{i j}^{2}\right) u^{\frac{-4}{n-2}} d v_{0}= & \int_{M} \frac{(\Delta u)^{2}}{u^{\frac{4}{n-2}}} d v_{0}+\frac{2(n+2)}{(n-2)^{2}} \int_{M} \frac{|\nabla u|^{4}}{u^{\frac{2 n}{n-2}}} d v_{0} \\
& -\frac{6}{n-2} \int_{M} \Delta u|\nabla u|^{2} u^{-\frac{n+2}{n-2}} d v_{0}-\int_{M} \sum B_{0 i j} u_{i} u_{j} u^{\frac{-4}{n-2}} d v_{0} \\
& -\frac{1}{n} \int_{M} s_{0}|\nabla u|^{2} u^{\frac{-4}{n-2}} d v_{0}
\end{aligned}
$$


Proof. By the Weitzenbock formula, we have

$$
\begin{aligned}
\frac{1}{2} \Delta|\nabla u|^{2}= & \sum u_{i j}^{2}+\sum(\Delta u)_{i} u_{i} \\
& +\sum B_{0 i j} u_{i} u_{j}+\frac{s_{0}}{n}|\nabla u|^{2} .
\end{aligned}
$$

Multiply both sides of equation (4) by $u^{\frac{-4}{n-2}}$ and integrate to get

$$
\begin{aligned}
\int_{M} \frac{1}{2} u^{\frac{-4}{n-2}} \Delta|\nabla u|^{2} d v_{0}= & \int_{M} u^{\frac{-4}{n-2}} \sum u_{i j}^{2} d v_{0}+\int_{M} u^{\frac{-4}{n-2}} \sum(\Delta u)_{i} u_{i} d v_{0} \\
& +\int_{M} \sum B_{0 i j} u_{i} u_{j} u^{\frac{-4}{n-2}} d v_{0}+\int_{M} \frac{s_{0}}{n} u^{\frac{-4}{n-2}}|\nabla u|^{2} d v_{0} .
\end{aligned}
$$

Use integration by parts to obtain

$$
\begin{aligned}
\frac{1}{2} \int_{M}|\nabla u|^{2}\left\{-\frac{4}{n-2} u^{-\frac{n+2}{n-2}} \Delta u+\frac{4(n+2)}{(n-2)^{2}} u^{\frac{-2 n}{n-2}}|\nabla u|^{2}\right\} d v_{0} \\
=\int_{M} u^{\frac{-4}{n-2}} \sum u_{i j}^{2} d v_{0}-\int_{M} u^{\frac{-4}{n-2}}(\Delta u)^{2} d v_{0}+\frac{4}{n-2} \int_{M} u^{-\frac{n+2}{n-2}} \Delta u|\nabla u|^{2} d v_{0} \\
\quad+\int_{M} u^{\frac{-4}{n-2}} \sum B_{0 i j} u_{i} u_{j} d v_{0}+\frac{s_{0}}{n} \int_{M} u^{\frac{-4}{n-2}}|\nabla u|^{2} d v_{0} .
\end{aligned}
$$

Simplify this to get Lemma 2.

Lemma 3. If $\int_{M} u^{\frac{2 n}{n-2}} d v_{0} \leq C_{1}$ and $n \geq 5$, then

$$
\int_{M}|\nabla u|^{4} u^{\frac{-2 n}{n-2}} d v_{0} \leq C_{3} \int_{M}(\Delta u)^{2} u^{\frac{-4}{n-2}} d v_{0}+C_{4}
$$

for some positive constants $C_{3}$ and $C_{4}$, depending only on $C_{1}, n$ and the geometry of $\left(M, g_{0}\right)$.

Proof. Consider

$$
h_{i j}=\varphi_{i j}-\varphi_{i} \varphi_{j}-\frac{1}{n}\left\{\Delta \varphi-|\nabla \varphi|^{2}\right\} g_{0 i j},
$$

where $\varphi=\frac{2}{n-2} \log u$. Thus

$$
\begin{aligned}
h_{i j}= & \frac{2}{n-2}\left\{\frac{u_{i j}}{u}-\left(1+\frac{2}{n-2}\right) \frac{u_{i} u_{j}}{u^{2}}\right\} \\
& -\frac{2}{n(n-2)}\left\{\frac{\Delta u}{u}-\left(1+\frac{2}{n-2}\right) \frac{|\nabla u|^{2}}{u^{2}}\right\} g_{0 i j} .
\end{aligned}
$$


Hence we have gotten

$$
\begin{aligned}
\sum h_{i j}^{2}= & \sum g_{0}^{i a} g_{0}^{j b} h_{i j} h_{a b} \\
= & \left(\frac{2}{n-2}\right)^{2}\left\{\sum u_{i j}^{2} u^{-2}+\left(\frac{n}{n-2}\right)^{2}|\nabla u|^{4} u^{-4}\right. \\
& \left.\quad-\frac{2 n}{n-2} \sum u_{i j} u_{i} u_{j} u^{-3}-\frac{1}{n}\left[(\Delta u) u^{-1}-\frac{n}{n-2}|\nabla u|^{2} u^{-2}\right]^{2}\right\} \\
=\left(\frac{2}{n-2}\right)^{2}\left\{\sum\right. & u_{i j}^{2} u^{-2}+\frac{n(n-1)}{(n-2)^{2}}|\nabla u|^{4} u^{-4} \\
& \quad-\frac{2 n}{n-2} \sum u_{i j} u_{i} u_{j} u^{-3}-\frac{1}{n}(\Delta u)^{2} u^{-2} \\
& \left.\quad+\frac{2}{n-2} \Delta u|\nabla u|^{2} u^{-3}\right\} .
\end{aligned}
$$

From equation (6), we have

$$
\begin{aligned}
A \equiv & \frac{(n-2)^{2}}{4} \int_{M}\left(\sum h_{i j}^{2}\right) u^{\frac{2(n-4)}{n-2}} d v_{0} \\
= & \int_{M} \sum u_{i j}^{2} u^{-2} u^{\frac{2(n-4)}{n-2}} d v_{0}+\frac{n(n-1)}{(n-2)^{2}} \int_{M}|\nabla u|^{4} u^{\frac{-2 n}{n-2}} d v_{0} \\
& -\frac{2 n}{n-2} \int_{M}\left[\sum u_{i j} u_{i} u_{j}\right] u^{-3} u^{\frac{2(n-4)}{n-2}} d v_{0}-\frac{1}{n} \int_{M} u^{\frac{-4}{n-2}}(\Delta u)^{2} d v_{0} \\
& +\frac{2}{n-2} \int_{M} \Delta u|\nabla u|^{2} u^{-3} u^{\frac{2(n-4)}{n-2}} d v_{0} \quad \text { by Lemma } 2 \\
= & \frac{n-1}{n} \int_{M}(\Delta u)^{2} u^{\frac{-4}{n-2}} d v_{0}+\frac{n^{2}+n+4}{(n-2)^{2}} \int_{M}|\nabla u|^{4} u^{\frac{-2 n}{n-2}} d v_{0} \\
& -\frac{2 n}{n-2} \int_{M}\left[\sum u_{i j} u_{i} u_{j}\right] u^{-\frac{n+2}{n-2}} d v_{0}-\frac{4}{n-2} \int_{M} \Delta u|\nabla u|^{2} u^{-\frac{n+2}{n-2}} d v_{0} \\
& -\int_{M} u^{\frac{-4}{n-2}} \sum B_{0 i j} u_{i} u_{j} d v_{0}-\frac{s_{0}}{n} \int_{M} u^{\frac{-4}{n-2}}|\nabla u|^{2} d v_{0} .
\end{aligned}
$$

Now apply integration by parts to obtain

$$
\begin{aligned}
\int_{M}\left[\sum u_{i j} u_{i} u_{j}\right] u^{-\frac{n+2}{n-2}} d v_{0} \\
=-\int_{M} \Delta u|\nabla u|^{2} u^{-\frac{n+2}{n-2}} d v_{0}-\int_{M}\left[\sum u_{i j} u_{i} u_{j}\right] u^{-\frac{n+2}{n-2}} d v_{0} \\
\quad+\frac{n+2}{n-2} \int_{M}|\nabla u|^{4} u^{\frac{-2 n}{n-2}} d v_{0}
\end{aligned}
$$

Therefore we have

$$
\int_{M}\left[\sum u_{i j} u_{i} u_{j}\right] u^{-\frac{n+2}{n-2}} d v_{0}=\frac{n+2}{2(n-2)} \int_{M}|\nabla u|^{4} u^{\frac{-2 n}{n-2}} d v_{0}-\frac{1}{2} \int_{M} \Delta u|\nabla u|^{2} u^{-\frac{n+2}{n-2}} d v_{0} .
$$


Substitute this equation into equation (7) to get

$$
\begin{aligned}
A= & \frac{n-1}{n} \int_{M}(\Delta u)^{2} u^{\frac{-4}{n-2}} d v_{0}+\frac{n^{2}+n+4}{(n-2)^{2}} \int_{M}|\nabla u|^{4} u^{\frac{-2 n}{n-2}} d v_{0} \\
& -\frac{n(n+2)}{(n-2)^{2}} \int_{M}|\nabla u|^{4} u^{\frac{-2 n}{n-2}} d v_{0}+\frac{n-4}{n-2} \int_{M} \Delta u|\nabla u|^{2} u^{-\frac{n+2}{n-2}} d v_{0} \\
& -\int_{M} u^{\frac{-4}{n-2}} \sum B_{0 i j} u_{i} u_{j} d v_{0}-\frac{s_{0}}{n} \int_{M} u^{\frac{-4}{n-2}}|\nabla u|^{2} d v_{0} \\
= & \frac{n-1}{n} \int_{M}(\Delta u)^{2} u^{\frac{-4}{n-2}} d v_{0}-\frac{n-4}{(n-2)^{2}} \int_{M}|\nabla u|^{4} u^{\frac{-2 n}{n-2}} d v_{0} \\
& +\frac{n-4}{n-2} \int_{M} \Delta u|\nabla u|^{2} u^{-\frac{n+2}{n-2}} d v_{0} \\
& -\int_{M} u^{\frac{-4}{n-2}} \sum B_{0 i j} u_{i} u_{j} d v_{0}-\frac{s_{0}}{n} \int_{M} u^{\frac{-4}{n-2}}|\nabla u|^{2} d v_{0} .
\end{aligned}
$$

Thus

$$
\begin{aligned}
\frac{n-4}{(n-2)^{2}} \int_{M}|\nabla u|^{4} u^{\frac{-2 n}{n-2}} d v_{0} \\
=-A+\frac{n-1}{n} \int_{M}(\Delta u)^{2} u^{\frac{-4}{n-2}} d v_{0}+\frac{n-4}{n-2} \int_{M} \Delta u|\nabla u|^{2} u^{-\frac{n+2}{n-2}} d v_{0} \\
\quad-\int_{M} u^{\frac{-4}{n-2}} \sum B_{0 i j} u_{i} u_{j} d v_{0}-\frac{s_{0}}{n} \int_{M} u^{\frac{-4}{n-2}}|\nabla u|^{2} d v_{0} \\
\leq-A+\frac{n-1}{n} \int_{M}(\Delta u)^{2} u^{\frac{-4}{n-2}} d v_{0} \\
\quad+\frac{n-4}{2(n-2)^{2}} \int_{M}|\nabla u|^{4} u^{\frac{-2 n}{n-2}} d v_{0}+2(n-4) \int_{M}(\Delta u)^{2} u^{\frac{-4}{n-2}} d v_{0} \\
\quad+\left(\beta_{1}-\frac{s_{0}}{n}\right) \int_{M}|\nabla u|^{2} u^{\frac{-4}{n-2}} d v_{0}
\end{aligned}
$$

where $\beta_{1}=\max _{x \in M}\left|B_{0 i j}\right|$.

Since $A \geq 0$, we thus obtain

$$
\begin{aligned}
& \frac{1}{2} \int_{M}|\nabla u|^{4} u^{\frac{-2 n}{n-2}} d v_{0} \\
& \leq\left\{4(n-2)^{2}+\frac{2(n-1)(n-2)^{2}}{n(n-4)}\right\} \int_{M}(\Delta u)^{2} u^{\frac{-4}{n-2}} d v_{0} \\
&+\epsilon\left|\beta_{1}-\frac{s_{0}}{n}\right|\left\{\frac{2(n-2)^{2}}{n-4}\right\} \int_{M}|\nabla u|^{4} u^{\frac{-2 n}{n-2}} d v_{0} \\
&+\frac{1}{\epsilon}\left|\beta_{1}-s_{0}\right|\left\{\frac{2(n-2)^{2}}{n-4}\right\} \int_{M} u^{\frac{2(n-4)}{n-2}} d v_{0} .
\end{aligned}
$$

If $\left|\beta_{1}-\frac{s_{0}}{n}\right|=0$, then we have nothing to worry about. If $\left|\beta_{1}-\frac{s_{0}}{n}\right| \neq 0$, then we 
can choose $\epsilon$ to be $\frac{(n-4)}{\left|\beta_{1}-\frac{s_{0} \mid}{n}\right|(n-2)^{2}}$. Thus we have obtained that

$$
\begin{aligned}
\frac{1}{2} \int_{M}|\nabla u|^{4} u^{-\frac{2 n}{n-2}} d v_{0} \\
\leq\left\{4(n-2)^{2}+\frac{2(n-1)(n-2)^{2}}{n(n-4)}\right\} \int_{M}(\Delta u)^{2} u^{\frac{-4}{n-2}} d v_{0} \\
\quad+\left|\beta_{1}-\frac{s_{0}}{n}\right|^{2} \frac{8(n-2)^{4}}{(n-4)^{2}}\left\{\int_{M} u^{\frac{2 n}{n-2}} d v_{0}\right\}^{\frac{n-4}{n}}\left\{\int_{M} d v_{0}\right\}^{\frac{4}{n}} \\
\leq\left\{4(n-2)^{2}+\frac{2(n-1)(n-2)^{2}}{n(n-4)}\right\} \int_{M}(\Delta u)^{2} u^{\frac{-4}{n-2}} d v_{0} \\
\quad+\left|\beta_{1}-\frac{s_{0}}{n}\right|^{2} \frac{8(n-2)^{4}}{(n-4)^{2}} C_{1}^{\frac{n-4}{n}} \operatorname{vol}^{\frac{4}{n}} \\
\equiv\left\{4(n-2)^{2}+\frac{2(n-1)(n-2)^{2}}{n(n-4)}\right\} \int_{M}(\Delta u)^{2} u^{\frac{-4}{n-2}} d v_{0}+\frac{1}{2} C_{4} .
\end{aligned}
$$

Multiply the above equation by 2 and set $2\left\{4(n-2)^{2}+\frac{2(n-1)(n-2)^{2}}{n(n-4)}\right\}=C_{3}$ to get Lemma 3.

Proof of Theorem 1. Apply Lemma 1 and Lemma 3 to get

$$
\int_{M}|\nabla u|^{4} u^{\frac{-2 n}{n-2}} d v_{0} \leq C_{5}
$$

where $C_{5}=2\left\{4(n-2)^{2}+\frac{2(n-1)(n-2)^{2}}{n(n-4)}\right\} C_{2}+C_{4}$. This is equivalent to

$$
\int_{M}\left|\nabla u^{\alpha_{1}}\right|^{4} d v_{0} \leq \frac{16(n-2)^{4}}{(n-4)^{4}} C_{5}
$$

where $\alpha_{1}=\frac{n-4}{2(n-2)}$.

Now set $\mu=2 \alpha_{1}$ to get

$$
\begin{aligned}
\int_{M}\left|\nabla u^{\mu}\right|^{2} d v_{0} & =\int_{M}\left|\nabla u^{2 \alpha_{1}}\right|^{2} d v_{0} \\
& =4 \int_{M}\left|\nabla u^{\alpha_{1}}\right|^{2} u^{2 \alpha_{1}} d v_{0} \\
& \leq 4\left\{\int_{M}\left|\nabla u^{\alpha_{1}}\right|^{4} d v_{0}\right\}^{\frac{1}{2}}\left\{\int_{M} u^{4 \alpha_{1}} d v_{0}\right\}^{\frac{1}{2}} .
\end{aligned}
$$

But $4 \alpha_{1}=\frac{2(n-4)}{n-2} \leq \frac{2 n}{n-2}$, thus we should have

$$
\int_{M} u^{4 \alpha_{1}} d v_{0} \leq C_{6}
$$

for some constant $C_{6}$. Thus we get

$$
\int_{M}\left|\nabla u^{\mu}\right|^{2} d v_{0} \leq C_{7}
$$


Now we also have

$$
\begin{aligned}
& \int_{M}\left(\Delta u^{\mu}\right)^{2} d v_{0} \\
&= 4 \int_{M}\left\{u^{\alpha_{1}}\left(\Delta u^{\alpha_{1}}\right)+\left|\nabla u^{\alpha_{1}}\right|^{2}\right\}^{2} d v_{0} \\
& \leq 8 \int_{M}\left(u^{\alpha_{1}}\right)^{2}\left(\Delta u^{\alpha_{1}}\right)^{2} d v_{0}+8 \int_{M}\left|\nabla u^{\alpha_{1}}\right|^{4} d v_{0} \\
&= 8 \int_{M}\left(u^{\alpha_{1}}\right)^{2}\left\{\alpha_{1} u^{\alpha_{1}-1} \Delta u+\alpha_{1}\left(\alpha_{1}-1\right) u^{\alpha_{1}-2}|\nabla u|^{2}\right\}^{2} d v_{0} \\
&+8 \int_{M}\left|\nabla u^{\alpha_{1}}\right|^{4} d v_{0} \\
& \leq 16 \alpha_{1}^{2} \int_{M} u^{4 \alpha_{1}-2}(\Delta u)^{2} d v_{0}+16 \alpha_{1}^{2}\left(\alpha_{1}-1\right)^{2} u^{4 \alpha_{1}-4}|\nabla u|^{4} d v_{0} \\
&+8 \int_{M}\left|\nabla u^{\alpha_{1}}\right|^{4} d v_{0} .
\end{aligned}
$$

Notice that

$$
4 \alpha_{1}-2=\frac{2(n-4)}{n-2}-2=-\frac{4}{n-2}
$$

and

$$
4 \alpha_{1}-4=\frac{2(n-4)}{n-2}-4=-\frac{2 n}{n-2} .
$$

After applying Lemma 1, (9) and (10), it is not too hard to see that there is a constant $C_{8}$ such that

$$
\int_{M}\left(\Delta u^{\mu}\right)^{2} d v_{0} \leq C_{8}
$$

Combining (11), (12) and (13), we conclude the proof of Theorem 1.

\section{Proof of Theorem 2}

As an application of our Theorem 1, we would like to prove Theorem 2 here. Thus one would like to use the spectrum to control the square integral of the scalar curvature. To this end, the main tool one could use is the heat invariants, i.e., the coefficients $a_{i}$ in the asymptotic expansion of the trace of the heat kernel

$$
Z(t)=\sum e^{-\lambda_{i} t} \simeq \frac{1}{(4 \pi t)^{\frac{n}{2}}} \sum a_{i} t^{i}
$$

as $t \rightarrow 0$. The coefficients $a_{i}$ are spectral invariants with the first few given by

\section{Lemma 4.}

$$
\begin{gathered}
a_{0}(g)=\operatorname{vol}(M, g) \\
a_{1}(g)=\frac{1}{6} \int_{M} s_{g} d v_{g} \\
a_{2}(g)=\frac{1}{180} \int_{M}\left[|W|^{2}+\frac{6-n}{n-2}|B|^{2}+\frac{5 n^{2}-7 n+6}{2 n(n-1)} s_{g}^{2}\right] d v_{g} .
\end{gathered}
$$


Proof. This is well known.

Now it is clear that when $n \leq 6$, each term in the expression of $a_{2}$ is positive. In particular, we have the square integral bound of scalar curvature while $\int_{M} u^{\frac{2 n}{n-2}} d v_{0}$ is exactly the volume of the new metric which has bound from $a_{0}$. Thus our Theorem 1 can be applied to this case.

Remark. If we make an assumption, we can get a further application of our Theorem 1:

Theorem 3. If $n \geq 7$ and $\left\{u_{i}^{\frac{4}{n-2}} g_{0}\right\}$ is an isospectral family with respect to both Laplace and conformal Laplace operators, then $\left\{u_{i}^{\mu}\right\}$ is a compact set in $H_{1}^{2}$ topology.

Proof. When we write down the third heat invariant $a_{2}$ with respect to both operators, we can see that their difference is the square integral norm of the scalar curvature. Volume bound comes out the same as above. Thus we still can use our Theorem 1 to get Theorem 3. More details about $a_{2}$ for a conformal Laplace operator may be found in [PR].

\section{ACKNOWLEDGMENT}

The author would very much like to thank Paul Yang for his interest.

\section{REFERENCES}

[An] M. Anderson, Remarks on the compactness of isospectral sets in lower dimensions, Duke Math. J. 63 (1991), 699-711. MR 92m:58140

[Au] T. Aubin, Nonlinear analysis on manifolds, Monge-Ampère equations, Springer-Verlag, New York, 1982. MR 85j:58002

[BGM] M. Berger, P. Gauduchon, and E. Mazet, Le spectre d'une varieté Riemannienne, Lecture Notes in Math., vol. 194, Springer-Verlag, New York, 1971. MR 43:8025

[B] A. Besse, Einstein manifolds, Springer-Verlag, New York, 1987. MR 88f:53087

[BCY] T. Branson, S.Y.A. Chang, and P.C. Yang, Estimates and extremals for zeta function determinants on four manifolds, Comm. Math. Phys. 149 (1992), 241-262. MR 93m:58116

[BG] R. Brooks and C. Gordon, Isospectral conformally equivalent Riemannian metrics, Bull. Amer. Math. Soc. (N.S.) 23 (1990), 433-436. MR 91a:58188

[BPY] R. Brooks, P. Perry, and P. Yang, Isospectral sets of conformally equivalent metrics, Duke Math. J. 58 (1989), 131-150. MR 90i:58193

[CY1] A. S. Y. Chang and P. Yang, Isospectral conformal metrics on 3-manifolds, J. Amer. Math. Soc. 3 (1990), 117-145. MR 91c:58140

[CY2] Compactness of isospectral conformal metrics on $S^{3}$, Comment. Math. Helv. 64 (1989), 363-374. MR 90c:58181

[C] J. Cheeger, Finiteness theorems for Riemannian manifolds, Amer. J. Math. 92 (1970), 61-74. MR 41:7697

[Ga1] Z. Y. Gao, Einstein metrics, J. Differential Geom. 32 (1990), 155-183.

[Ga2] Convergence of Riemannian manifolds, Ricci and $L^{\frac{n}{2}}$ curvature pinching, J. Differential Geom. 32 (1990), 349-381.

[GW] R. Greene and H. Wu, Lipschitz convergence of Riemannian manifolds, Pacific J. Math. 139 (1988), 119-141. MR 89g:53063

[GLP] M. Gromov, J. Lafontaine, and P. Pansu, Structures metriques pour les varietes Riemanniennes, Cedic-Fernand/Nathan, Paris, 1981.

[G] M. Gursky, Compactness of conformal metrics with integral bounds on curvature, thesis, Cal Tech., 1991.

[Mo] M. Min-Oo, Almost Einstein manifolds of negative Ricci curvature, J. Differential Geom. 32 (1990), 457-472. MR 91g:53047

[P] S. Peter, Convergence of Riemannian manifolds, Compositio Math. 62 (1987), 3-16. 
[PR] T. Parker and S. Rosenberg, Invariants of conformal Laplacians, J. Differential Geom. 25 (1988), 199-222. MR 89e:58118

[X] X. Xu, On compactness of isospectral conformal metrics of 4-manifolds, Nagoya Math. J. (to appear).

Department of Mathematics, National University of Singapore, Singapore 0511

E-mail address: matxuxw@leonis.nus.sg 